\title{
Noise elimination in lead-slowing down neutron spectrometer using digital signal processing
}

\author{
T. OISHI*, T. YAMAUCHI, M. HAGIWARA, M.BABA \\ Cyclotron Radioisotope Center, Tohoku University \\ 6-3, Aoba, Aramaki, Aoba, Sendai, Miyagi, Japan \\ E-mail:oishit@cyric.tohoku.ac.jp, yamat@cyric.tohoku.ac.jp, \\ hagi@cyric.tohoku.ac.jp, babam@cyric.tohoku.ac.jp

\section{H.YUKI, T. OTSUKI} \\ Laboratory of Nuclear Science, Tohoku University \\ 1-2-1, Mikamine, Taihaku, Sendai, Miyagi, Japan \\ E-mail: hideyuki@LNS.tohoku.ac.jp, ohtsuki@LNS.tohoku.ac.jp
}

\section{J. HORI}

Research Reactor Institute, Kyoto University

2, Asayonishi, Kumatori, Sennan, Osaka, Japan

E-mail: hori@rri.kyoto-u.ac.jp

In a fission cross-section measurement with a lead-slowing down neutron spectrometer the neutron energy range was extended to higher energy by applying a digital signal processing (DSP) technique. With this technique, it is possible to eliminate large noise signals which are synchronized with the accelerator pulse and disturb the measurement in the high energy region. Furthermore, with this technique the timing signal is obtained with a short delay from the beam burst. Consequently, the high energy range could be extended to the $\mathrm{MeV}$ region (from several $\mathrm{keV}$ with the previous technique).

International Workshop on Fast Neutron Detectors University of Cape Town, South Africa April 3-6, 2006

\footnotetext{
${ }^{*}$ Speaker
} 


\section{Introduction}

Fission cross sections of minor actinides (MA) are very important for the design of an accelerator driven transmutation system (ADS). However, there are significant differences between the evaluated nuclear data libraries. The main cause of these discrepancies is the lack of good experimental data due to the difficulty to obtain high quality MA samples and the large background due to $\alpha$ particles. To improve the data basis the fission cross section of MA should be measured with high accuracy. A lead-slowing-down spectrometer enables this kind of measurement, due to its capability to operate at very high neutron flux as compared to a time-offlight spectrometer.

Kobayashi et al. have already provided high quality experimental data using Kyoto universities lead-slowing-down spectrometer (KULS) $[1,2,3]$. In KULS, the neutron flux is about 1000 times higher than in comparable experiments with TOF method. On the other hand the energy resolution of KULS is inferior (about $40 \%$ [4] compared to $3 \%$ with TOF. Besides that, the measurement with KULS is possible with a small amount of sample material.

However, the high energy end was restricted to about $10 \mathrm{keV}$ in the past measurement. Because large noise synchronized with the accelerator pulse was overlapping the signal and disturbed the measurement of high energy events. Some suppression of noise was observed by electromagnetic shielding with an aluminum foil wrapped around the preamplifiers and the cables, but it was not sufficient to significantly extend the energy range.

The digital signal processing (DSP) technique which samples the waveform of the detector signal directly, converts it to digital data and enables its analysis on a computer provides the means to eliminate the noise. DSPs proved to be very useful in our experiment with the Bragg curve counter [5] and the high count rate measurement. In this work, the DSP technique was applied to KULS in order to extend the energy range.

\section{Digital signal processing}

The digital signal processing (DSP) technique enables us to analyze the signals flexibly and readily, because the full signal waveform from a detector is acquired as digital data, and information about the detected radiation, for example its energy and arrival time, can be derived through the analysis of the signal waveform using computers. This method has been applied since many years, but it has not been always useful due to slow ADCs and computers. Recently, signal waveform acquisition became possible with reasonable counting rate in relatively simple way owing to the increasing speed of ADCs and computers. Therefore it becomes possible to apply the DSP technique efficiently to the practical radiation detection.

In general, the DSP technique is very powerful tool for the radiation measurement. The most significant advantage of the DSP technique is the possibility of a flexible signal analysis which is impossible with the analog circuits. In addition, the DSP technique has more advantages, like reduction of the load for preparation of experiments owing to very few analog circuits required, and the possibility of high quality data analysis by signal processors and fast computers

\section{Experiment}

\subsection{Experimental arrangement}

The experiment was carried out with the Kyoto university lead-slowing-down spectrometer driven by a $46 \mathrm{MeV}$ electron linear accelerator. The lead-slowing-down 
spectrometer is based on the fact, that the average energy of neutrons can be determined by that the slowing down time of fast neutrons in matter. The structure of KULS is shown in Fig. 1. An air-cooled tantalum target which is set in the center of a $1.5 \mathrm{~m}$ lead cube is irradiated by the pulsed beam of the $46 \mathrm{MeV}$ electron liniac to generate neutrons. The neutrons lose their energies by elastic collisions with lead. The average energy $E$ of the neutrons is related to the slowing down time $\mathrm{t}$ by the relationship $\mathrm{E}=\mathrm{K} /\left(\mathrm{t}+\mathrm{t}_{0}\right)^{2}$, where $\mathrm{K}$ is a constant and $\mathrm{t}_{0}$ is a correction constant.

A back-to-back type fission chamber is placed in the experimental hole, which is covered by a bismuth plate to reduce high energy $\gamma$ rays from lead. This fission chamber detects the fission fragments of the fission reactions induced by the

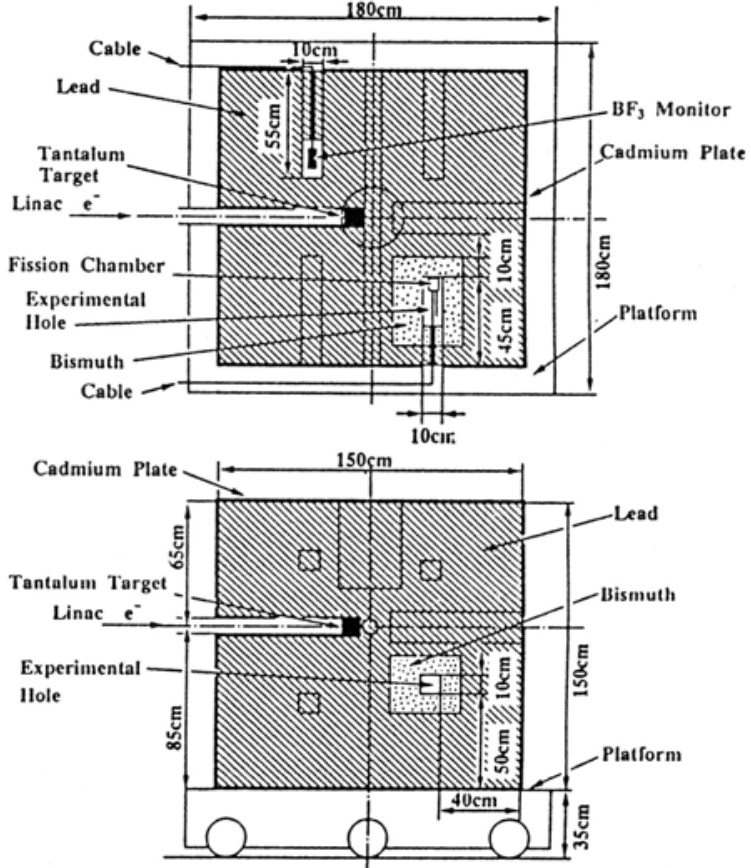

Figure 1: The structure of KULS slowed down neutrons. In this work a foil with electrodeposited ${ }^{235} \mathrm{U}$, whose fission cross section is well known, was put on one side and a ${ }^{237} \mathrm{~Np}$ layer was deposited on the other side of the fission chamber electrodes. The signals from both chambers were connected to charge sensitive preamplifiers via $\sim 1.5 \mathrm{~m}$ long coaxial cables. The preamplifiers and the cables were wrapped with aluminum foils for electromaganetic shielding. This shielding reduced significantly the pick-up noise, induced by the so called "gamma-flash", which is synchronized with the electron beam pulse hitting the target. This "gamma-flash" significantly disturbed the measurement in high energy region with the previously used analogue readout technique.

\subsection{Data acquisition}

Figure 2 shows the schematics of the data acquisition system which was used. The signals of the fission chamber were fed into the charge sensitive preamplifiers (ORTEC/142PC) and the tails of the output pulses were cut off by timing filter amplifiers. Then the output signal waveform was acquired by a digital storage oscilloscope (DSO, LeCroy Wavepro7000). The amplitude resolution is 8 bits and the maximum sampling rate is $10 \mathrm{GS} / \mathrm{sec}$. By the DSO the signal waveform was converted into digital data and stored in the HDD (hard disc drive) of the DSO.

Another computer was connected to the DSO via Ethernet, and online analysis was carried out on this computer. Using this online analysis, the results could be checked immediately without reducing the acquisition speed of the scope. In this work, the online analysis was used to obtain a two-dimensional scatter plot of the

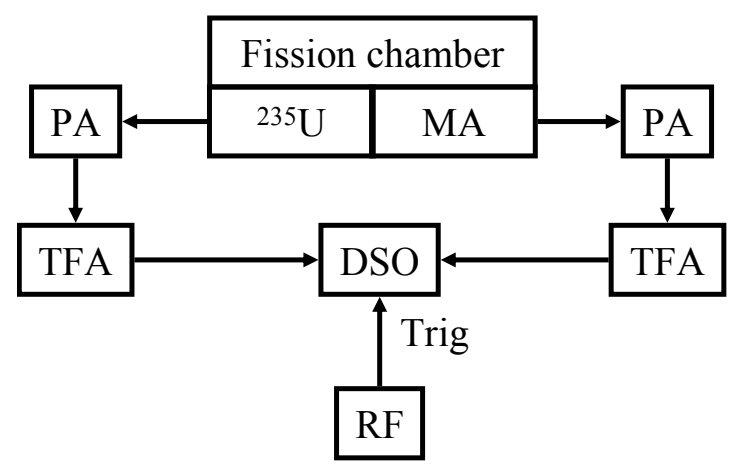

Figure 2: Data acquisition system with DSP, where PA: Preamplifier, TFA: Timing filter amplifier, DSO: Digital storage oscilloscope, RF: RF signal of accelerator 
pulse-height versus the slowing down time. The RF signal of the accelerator was used as a trigger signal. The signal waveform was acquired for $45 \mu \mathrm{sec}$ (corresponds to $\sim 94 \mathrm{eV}$ in neutron energy) after the trigger. The typical signal waveform is shown in Fig. 3. The horizontal axis is set to 0.02 $\mu \mathrm{sec} / \mathrm{ch}$. The RF signal was delayed by a gate and delay generator. In this figure we observe the noise which is synchronized with the RF signal. This noise prevented fission fragment signals to be detected properly. In this figure, there are two

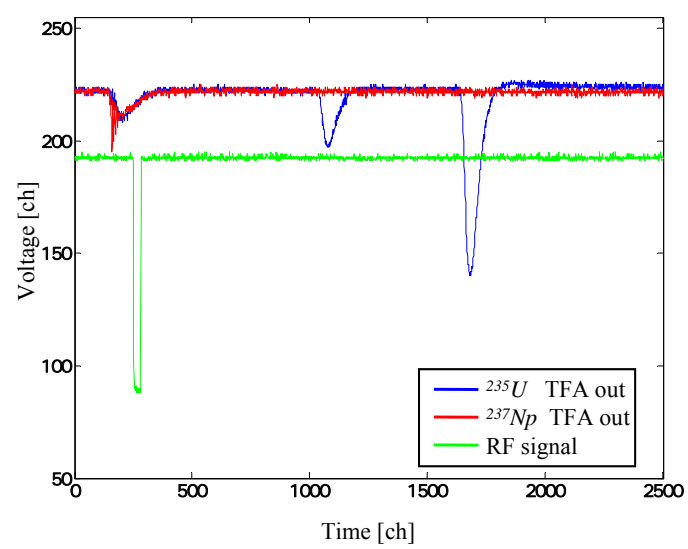

Figure 3: Signal waveform of the TFA output and RF signal. signals from fission fragments in the same frame. This demonstrates that the measurement of slowing down time requires multi-stop capability of online analysis.

\section{Data analysis technique}

\subsection{Noise reduction}

The typical shape of the electromagnetic pickup signal is shown in Fig. 4. Through the observation of many events, we found that the shape and size of the pickup signal is almost constant. Therefore, the pickup signal could be eliminated by subtracting a representative waveform, which was obtained from the average of ten pure pickup pulse tails which did not contain contributions of fission fragments. An example of the result of this procedure is shown in Fig. 5 (the polarity of signal is inverted from Fig.4). The pickup signal was almost completely removed, and the fission fragment's signal can now be clearly recognized. Using this method, also the signal-starting time can now be accurately determined, thus the measurable energy range is extended towards higher energies regions.

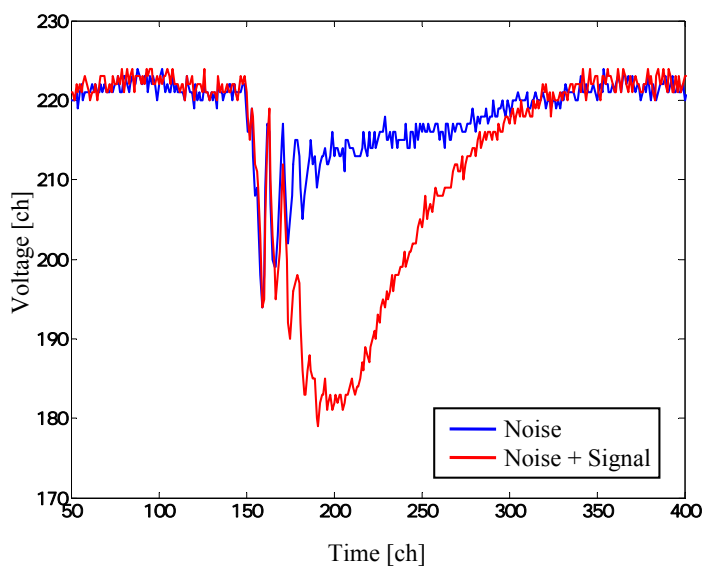

Figure 4: Typical waveform of a detected fission fragment, distorted by an electromagnetic pick up signal. .

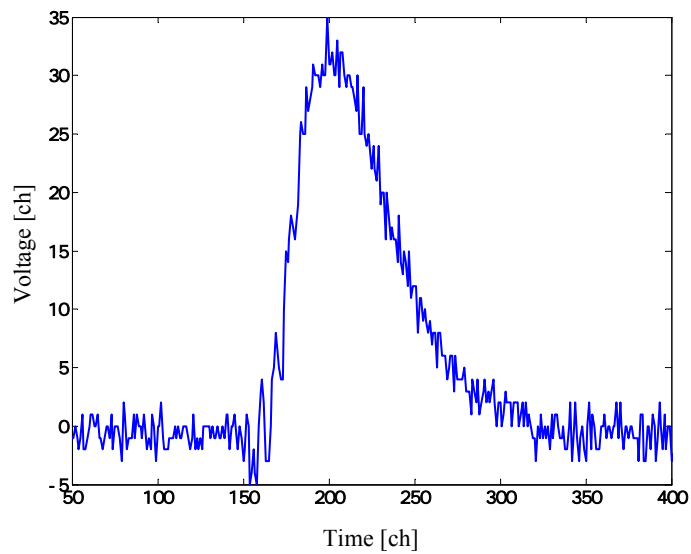

Figure 5: The waveform of a detected fission fragment after elimination of electromagnetic pick up signal. 


\subsection{Determination of slowing-down time}

At high neutron energies, small uncertainties in the timing measurements introduce large uncertainties in the energy scale, namely the region of short slowing down time. For example, 1 $\mathrm{MeV}$ corresponds to $0.44 \mu \mathrm{sec}$ in slowing down time. In this case very good time resolution is required. The energy calibration is difficult because no resonance filter is available in the high energy region. For this reason, a time measurement technique which can detect the "start-of-the-rise" of the pulse is necessary.

In this work, a new method which solves this problem is presented. In the recorded pulse trail the last intersection point between the baseline and the signal waveform in front of the peak is detected as the "start-of-the rise" and named as "baseline method" (see Fig. 6). With this baseline method, it is possible to detect the start of the rise while the error is larger and the timing resolution is worse than with the CFD (constant fraction discriminator) method. This difference in the error is not large: for example, the error in

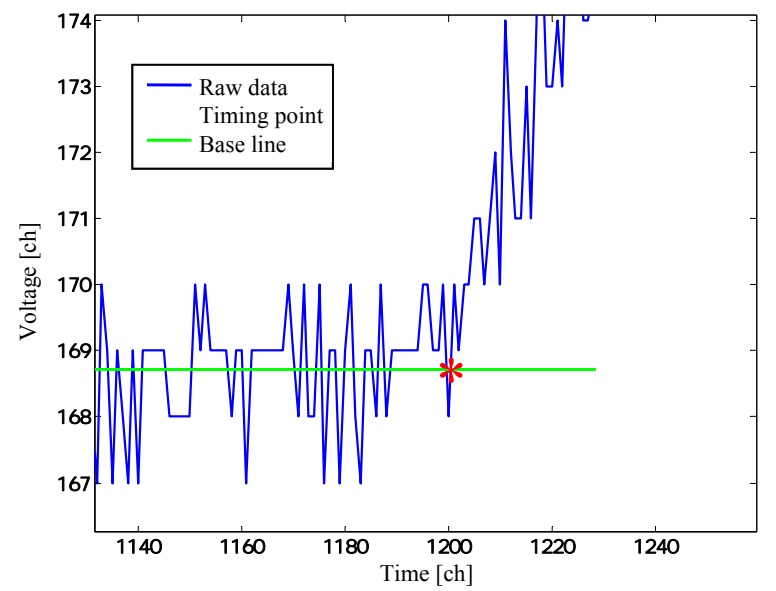

Figure 6: Fraction of a fission fragment pulse trail. The "start of the rise" was detected .and is marked with "*". energy measurement at $1 \mathrm{MeV}$ is $24.6 \%$ by the baseline method and $19.5 \%$ by the CFD method. The importance of this error is low because the resolution of the spectrometer is anyway not better than $40 \%$. But the time shift by the CFD results in a difference of $\sim 50 \%$. Detection of start-of-the rise is more important than error and resolution, accordingly the baseline method is effective in the high energy region.

\section{Experimental results for the ${ }^{237} \mathrm{~Np}$ fission cross section}

The experimental result obtained for the fission cross section of ${ }^{237} \mathrm{~Np}$ is shown in Fig. 7. These values were derived by using the JENDL-3 data for ${ }^{235} \mathrm{U}$ and by binning the data in energy bins of $40 \%$ width. It is clear that the DSP technique could extend the upper limit into the $\mathrm{MeV}$ region. Therefore, the effectiveness of the DSP technique is apparent while further studies are needed for a proper calibration of the energy scale. Below $\sim 0.5 \mathrm{keV}$, the present result is in good agreement with data of Kobayashi et al.

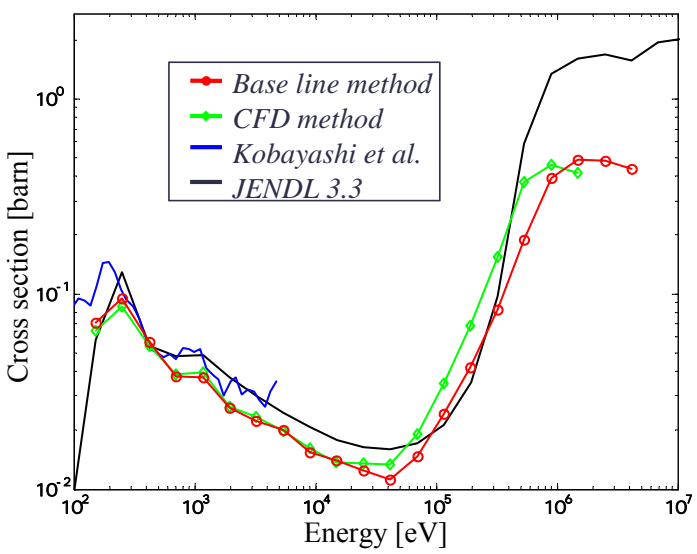

Figure 7: The result of fission cross section of

${ }^{237} \mathrm{~Np}$. The measurements using DSP technique are baseline method and d-CFD method. 
there is a small difference between the DSP results and JENDL in the absolute value, while the shape is in good agreement. Besides, the shapes don't agree above $40 \mathrm{keV}$, although they show the same tendency that the cross section rise steeply above $100 \mathrm{keV}$. The reason for the difference may be attributed to the fact that the energy resolution of the KULS is less than $40 \%$ and the relationship between the energy and the slowing down time is not properly assigned in high energy region. Therefore, we are promoting Monte Carlo simulation to clarify the problem, and also the revision of the data processing to take into account the cross section shape into the data reduction.

There is a difference in a region above $40 \mathrm{keV}$ between the timing by the baseline method and by the d-CFD method which is simply constant fraction discrimination using the DSP technique. In d-CFD method, the high energy events are shifted to the lower energy side because the d-CFD method derived the timing at a later time than the "start of the rise" of the pulse. With considering the principle of the timing detection, it is clear that the baseline method will be superior.

\section{Summary}

The DSP technique was introduced in order to overcome the problem that the measurement of the fission cross section above about $10 \mathrm{keV}$ was impossible in the previous experiment using KULS $[1,2,3]$. A DSO was used as a data taking system with an online analysis. Electromagnetic pick up signals, which were responsible for the rather low energy limit, were eliminated using the DSP technique in addition to proper electromagnetic shielding of the electronic modules. Thus, the signals of fission fragments could be clearly recognized up to high neutron energies. Besides, a baseline method was developed in order to detect the high energy event with larger precision. Using these methods, the fission cross section of ${ }^{237} \mathrm{~Np}$ was measured, although more investigation was needed. The DSP technique has potential of applicability to various purposes.

\section{Acknowledgement}

The author wish to express our gratitude to Drs. S. Yamamoto and K. Kobayashi for their invaluable help to the experiment.

\section{Reference}

[1] A. Yamanaka et al., J. Nucl. Sci. Technol., 30, 9, 863-869 (1993)

[2] S. Yamamoto et al., Nucl. Sci. Eng., 126, 201-212 (1997)

[3] T. Kai et al., Ann. of Nucl. Energy 28,723-739 (2001)

[4] R. E. Slovacek et al., Nucl. Sci. Eng., 62, 455 (1977)

[5] M. Hagiwara et al., Proceedings of American Institute of Physics 769, 1031-1034 (2004) 